\title{
Carotid intima-media thickness and its correlation with anthropometric and clinical variables in pediatric patients with obesity: An exploratory study
}

\author{
Erika L. Alba-Rojas, Julieta Rodríguez-de Ita, Jesús M. Yañez-Sánchez, Marcelo Salán-Gómez, \\ Marcos A. Acosta-Sandoval, Ricardo J. Estrada-Mendizábal, Margarita Riojas-Barrett and \\ Gabriela Guzmán-Navarro* \\ Escuela de Medicina y Ciencias de la Salud, Monterrey, Tecnologico de Monterrey, Nuevo Leon, Mexico
}

\begin{abstract}
Aim: Mexico is currently ranked first in childhood obesity. Measurement of carotid intima-media thickness (cIMT) is an important diagnostic method to identify patients at risk of or already having frank atherosclerosis. The aim of this study was to determine if there was a correlation between cIMT and anthropometric and clinical variables in pediatric patients with obesity. Methods: Anthropometric and clinical variables were collected from 28 pediatric patients with obesity between 10 and 18 years old who underwent measurement of cIMT. cIMT was measured using a Doppler ultrasound with a $7 \mathrm{MHz}$ transducer. All measurements were made with the same Philips HD (Mode B) ultrasound by the same pediatric cardiologist. Statistical analysis was performed using the Spearman correlation coefficient and linear regression. Results: There was a significant correlation between the cIMT and both body mass index (BMI) and triceps skinfold thickness, but not with leptin, fasting glucose, or cholesterol levels. cIMT was $\geq 0.40 \mathrm{~mm}$ in 24 (85\%) patients. Pre-hypertension was found in 16 (57\%) and hypertension in 4 (14\%). Cholesterol levels > $200 \mathrm{mg} / \mathrm{dL}$ were found in 6 (21\%). clMT was positively correlated with weight $\left(r_{s}=0.412, p=0.030\right)$, BMI (coefficient $r_{s}$ of 0.452 and $p=0.016$ ), and triceps skinfold (coefficient $r_{s}$ of 0.396 and $p=0.037)$. Conclusions: Anthropometric measurements, including BMI and triceps skinfold thickness, should be used in the evaluation of the cardiovascular risk of the pediatric population with obesity.
\end{abstract}

Key words: Carotid intima-media thickness. Mexico. Obesity. Pediatric.

\section{Introduction}

Obesity is a significant public health problem worldwide. Its incidence has increased exponentially along with many of the complications associated with it, including diabetes mellitus, arterial hypertension, and dyslipidemias ${ }^{1}$. Recent statistics show that Mexico ranks second in the prevalence of worldwide obesity in adults and first in childhood obesity ${ }^{2,3}$. According to a national survey on health and nutrition in 2016 (ENSANUT, initials in Spanish), the prevalence of obesity among children in Mexico aged 5-11 was $33.2 \%$. Among girls, $20.6 \%$ were overweight and $12.2 \%$ were obese. Among boys, $15.4 \%$ were overweight and $18.6 \%$ were obese. The incidence, particularly of obesity, is slightly higher in males than that in females ${ }^{4}$.

Coronary heart disease was initially considered to occur exclusively in adulthood. However, evidence of early vascular changes associated with it has been found in obese children, including increased right atrial

\section{Correspondence:}

*Gabriela Guzmán-Navarro

E-mail: gabriela.guzman @tec.mx
Available online: 30-09-2019

Date of reception: 27-02-2019

Date of acceptance: 03-06-2019

DOI: 10.24875/RMU. 19000071
Medicina Universitaria. 2019;21(3):100-104 www.medicinauniversitaria.org CC BY-NC-ND license (http://creativecommons.org/licenses/by-nc-nd/4.0/). 
diameter, arterial stiffness, and thickening of the carotid artery intima and media5,6. Measurement of the thickness of these layers of the carotid arterial wall is performed with a B-mode ultrasound. As the term carotid intima-media thickness (cIMT) implies, this measure takes into account the thickness of the tunica intima and tunica media of the artery. Pathological thickening may indicate hypertrophy of the smooth muscle of the artery (tunica media) or accumulation of lipid products in the subintima area. It is not possible to identify precisely which of these areas is thickened in any particular case. Early changes occur predominantly in the tunica media and later in the intima. Either way, an increased cIMT reflects increased cardiovascular risk ${ }^{7-9}$.

Coronary angiography is the gold standard for the diagnosis of coronary heart disease but is an expensive and invasive procedure. A strong correlation has been demonstrated between coronary and carotid atherosclerosis, which explains why cIMT is a good predictor of coronary heart disease ${ }^{10}$. Ultrasound measurement of cIMT is widely performed, as it is economical, widely available, and noninvasive, although it requires trained personnel to perform it. The average cIMT in both sexes at the age of 6 is 0.37 , and by the age of 18 , it increases to 0.39 in women and 0.41 in men $^{11}$.

There is a growing interest in the use of cIMT to identify early evidence of atherosclerosis, and therefore, to make an early assessment of the risk of cardiovascular disease in children. Studies in children with obesity have yielded findings similar to those in adults. This study of obese children aimed to evaluate the anthropometric and clinical parameters that might correlate with an increased cIMT.

\section{Patients and methods}

This was an observational, cross-sectional study conducted from July to October 2014. It was approved by the Ethics Committee of the Escuela de Medicina y Ciencias de la Salud del Tecnológico de Monterrey.

Patients were recruited from those seen at the obesity clinic in the Centro de Atención Médica Santos y De La Garza Evia, in the city of Santa Catarina, Nuevo León. Inclusion criteria were ages 10-18 and the diagnosis of obesity made by a physician based on a body mass index (BMI) above the $>95^{\text {th }}$ percentile for age and sex, according to the Centers for Disease Control and Prevention $(C D C)$ guidelines. Children meeting the criteria were invited to participate in the study protocol. Parents signed a consent form, and the minors were asked to sign an assent form. Exclusion criteria included a history of diabetes, hypothyroidism, Cushing's syndrome, or other endocrinological diseases, as well as the use of antihypertensive or hypoglycemic medications or steroids. Participants were suspended from the study if any of the exclusion criteria were detected after entry or if the parents or patients decided not to continue.

Anthropometric variables included the following. Height was measured in meters using a retractable tape measure. Patients were measured standing upright and facing forward without shoes. Weight was measured in kilograms using a Tanita BC 689 scale. Obesity was defined as a $\mathrm{BMI} \geq 95^{\text {th }}$ percentile for age according to the CDC guidelines. BMI was calculated as weight in kilograms divided by the square of height in meters. The triceps skinfold was measured with a Seca plicometer. The adipose tissue was palpated with the examiner's left thumb and forefinger, excluding the muscle and was measured with the plicometer in the right hand. Results were classified according to percentiles proposed by a committee of the World Health Organization ${ }^{12}$.

Arterial blood pressure was measured according to the guidelines of the American Academy of Pediatrics (AAP) (child sitting quietly for $5 \mathrm{~min}$, and seated with his or her back supported, feet on the floor and right arm supported, cubital fossa at heart level) by auscultation with a wall-mounted sphygmomanometer using a pediatric cuff and a pediatric stethoscope. The pressure was classified as normal if below the $90^{\text {th }}$ percentile, pre-hypertension if between the $90^{\text {th }}$ and $95^{\text {th }}$ percentiles, and hypertension if above the $95^{\text {th }}$ percentile ${ }^{13}$.

cIMT was measured using a Doppler ultrasound with a $7 \mathrm{MHz}$ transducer. cIMT was measured in both the right and left carotid arteries, and the mean of the two measurements was recorded. All measurements were made with the same Philips HD (Mode B) ultrasound by the same pediatric cardiologist. It should be mentioned that the cardiologist who made the measurements was not blinded. Values were reported in $\mathrm{mm}$, with a thickness of $\leq 0.39 \mathrm{~mm}$ considered normal and those $>0.39 \mathrm{~mm}$ abnormal, having $0.40 \mathrm{~mm}$ as the cutoff value ${ }^{7}$ (Fig. 1).

Biochemical tests included leptin, glucose, and cholesterol levels. Plasma leptin was measured using an enzyme-linked immunosorbent assay (ELISA) kit (Anti-Leptin RD systems, Cat No. DLP00), standardized with inner control and cohort values established by the manufacturer. The mean value of three tests was recorded. Normal levels were $4.73 \pm 0.69 \mathrm{ng} / \mathrm{mL}^{14}$. Serum glucose was measured by enzymatic assay, and values between 60 and $100 \mathrm{mg} / \mathrm{dL}$ were considered normal according to the AAP ${ }^{15}$. Cholesterol was measured 


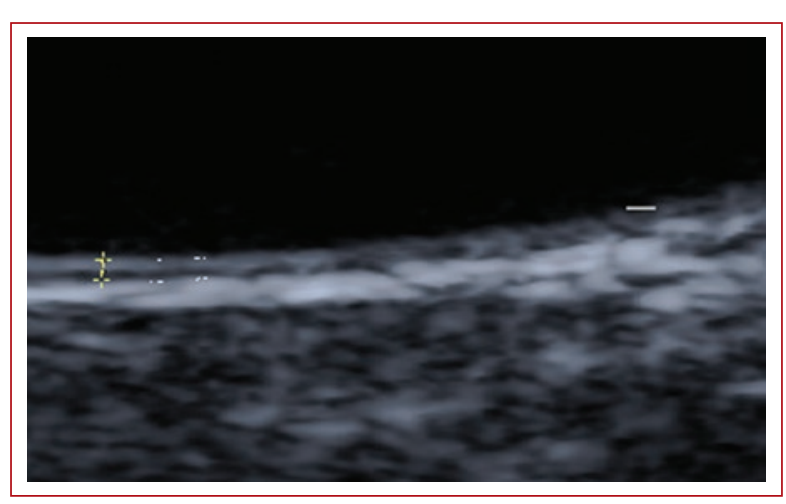

Figure 1. Carotid intima-media thickness (cIMT) measured using Doppler ultrasound with a $7 \mathrm{MHz}$ transducer. cIMT was measured in both the right and left carotid arteries, and the mean of the two measurements was recorded. All measurements were made with the same Philips HD (Mode B) ultrasound.

using serum enzymatic assay, and values of total cholesterol $<170 \mathrm{mg} / \mathrm{dL}$ were considered normal, values of $170-199 \mathrm{mg} / \mathrm{dL}$ borderline and values $\geq 200 \mathrm{mg} / \mathrm{dL}$ elevated according to the AAP guidelines ${ }^{16}$. Laboratory analysis was performed in the Cardiology Department of the Centro Médico Zambrano Hellion and in the Laboratory of Biotechnology of the Instituto Tecnológico y de Estudios Superiores de Monterrey.

Data were recorded in an Excel 2010 database and analyzed with IBM SPSS Statistics 21. Traditional descriptive statistics (central tendency, dispersion, and position) were reported for quantitative values, and frequencies were used for qualitative values. Correlations and associations were made using the Spearman test.

\section{Results}

A total of 28 patients were recruited, 17 boys and 11 girls (Table 1). cIMT was $\geq 0.40 \mathrm{~mm}$ in $24(85 \%)$ patients. Pre-hypertension was found in $16(57 \%)$, and hypertension in $4(14 \%)$. Cholesterol levels > $200 \mathrm{mg} / \mathrm{dL}$ were found in $6(21 \%)$.

Spearman rank correlation analysis was performed comparing cIMT with each variable (Table 2). cIMT was positively correlated with weight $\left(r_{s}=0.412, p=0.030\right)$, BMI (coefficient $r_{s}$ of 0.452 and $p=0.016$ ), and triceps skinfold (coefficient $r_{s}$ of 0.396 and $p=0.037$ ).

On linear regression, the dependent variable was cIMT, and the independent variables were BMI, waist-to-height ratio, triceps skinfold, and body fat percentage. The executed linear regression model was statistically significant with a value of $p=0.027$, accounting for $37 \%$ of the variance $\left(R^{2}=0.37\right)$. We observed in our model that the
Table 1. Anthropometric and clinical variables of children with obesity

\begin{tabular}{|l|c|}
\hline Variables & $\mathbf{n}=\mathbf{2 8}$ \\
\hline Age, years & $11(1)$ \\
\hline Gender, M:F & $17: 11$ \\
\hline Height, m & $1.52 \pm 0.09$ \\
\hline Weight, kg & $59.9(19.7)$ \\
\hline BMI, kg/m² & $27.75(5.7)$ \\
\hline BMI, percentiles & $97(2)$ \\
\hline Body fat, \% & $37.6(6.2)$ \\
\hline Waist circumference, cm & $91.5(11.5)$ \\
\hline Waist-to-height ratio & $0.60 \pm 0.06$ \\
\hline Triceps skinfold, cm & $27.5(12)$ \\
\hline clMT, mm & $0.43(0.05)$ \\
\hline Systolic blood pressure, mm Hg & $110(20)$ \\
\hline Diastolic blood pressure, mm Hg & $68.6 \pm 11.3$ \\
\hline Systolic blood pressure percentile & $50(40)$ \\
\hline Diastolic blood pressure percentile & $50(40)$ \\
\hline Triglycerides, mg/dL & $128(73)$ \\
\hline Glucose, mg/dL & $86 \pm 9.2$ \\
\hline Total cholesterol, mg/dL & $174.04 \pm 40.6$ \\
\hline HDL, mg/dL & $43.88 \pm 10.1$ \\
\hline LDL, mg/dL & $102.5 \pm 36.7$ \\
\hline Leptin, ng/dL & $15.8 \pm 0.6$ \\
\hline (n) & \\
\hline
\end{tabular}

BMI: body mass index; cIMT: carotid intima-media thickness; HDL; high-density lipoprotein; LDL: low-density lipoprotein. Data are expressed as a mean \pm standard deviation or median (interquartile range).

factors most predictive of cIMT were BMI and the triceps skinfold, with $p$ values of 0.03 and 0.37 , respectively.

\section{Discussion}

We investigated a possible correlation between cIMT and anthropometric and clinical variables in children with obesity. The median cIMT in our sample, $0.42 \mathrm{~mm}$, was similar to values previously described in the literature ${ }^{5,6}$. As we only studied patients with obesity, we expected that most would have a cIMT $\geq 0.4 \mathrm{~mm}$, and found this indeed to be true in $24(85 \%)$ of our patients. This is of paramount clinical importance since cIMT is an early marker of cardiovascular disease ${ }^{6-8}$. Given that the thickening of the arterial wall can be identified during the initial phases of atherosclerosis before the arterial 
Table 2. Spearman correlation coefficients between anthropometric and clinical variables and cIMT in children with obesity

\begin{tabular}{|l|c|c|}
\hline Variables & $\begin{array}{c}\text { Spearman correlation } \\
\text { coefficient }\end{array}$ & p value \\
\hline Age & 0.162 & 0.412 \\
\hline Weight & 0.412 & $0.030^{*}$ \\
\hline BMI & 0.452 & $0.016^{*}$ \\
\hline BMI percentiles & 0.174 & 0.375 \\
\hline Systolic blood pressure & 0.003 & 0.990 \\
\hline percentile & 0.116 & 0.555 \\
\hline $\begin{array}{l}\text { Diastolic blood pressure } \\
\text { percentile }\end{array}$ & & 0.525 \\
\hline Waist & 0.125 & 0.439 \\
\hline Waist-to-height ratio & -0.152 & 0.716 \\
\hline Body fat percentage & 0.072 & $0.037^{*}$ \\
\hline Triceps skinfold & 0.396 & 0.611 \\
\hline Triglycerides & -0.101 & 0.094 \\
\hline Glucose & -0.322 & 0.157 \\
\hline HDL & 0.275 & 0.881 \\
\hline LDL & 0.03 & 0.912 \\
\hline Total cholesterol & -0.021 & 0.103 \\
\hline Leptin & 0.315 & \\
\hline & & \\
\hline
\end{tabular}

BMI: body mass index; cIMT: carotid intima-media thickness; HDL: high-density lipoprotein; LDL: low-density lipoprotein. ${ }^{*}$ Statistically significant values $(p<0.05)$.

lumen becomes markedly compromised, the evaluation of cIMT by ultrasound represents a suitable measure that depicts a direct incidence of atherosclerosis in all its clinical forms ${ }^{17}$, even from the subclinical stages ${ }^{18}$. After our study, the patients with abnormally high cIMT were followed by pediatric consultants and underwent echocardiography. In this pediatric sample, therefore, obesity was associated with a high risk of abnormal cIMT. There were also substantial numbers of patients with pre-hypertension, hypertension, and high cholesterol levels. Furthermore, the association between hypertensive states (represented by a percentile $>90$ according to age, height, and gender) and its relation with an increased cIMT, as well as arterial stiffness in patients with cardiovascular risk factors such as obesity or metabolic syndrome, is supported by similar studies among the young ${ }^{19,20}$. However, such features are not universal criteria present among all pediatric populations with obesity. Thus, clinical assessment should focus on a wide range of physical parameters.
We found a significant relationship between the triceps skinfold and cIMT on both correlation and linear regression analyses. The study, therefore, suggests that the anthropometric parameters of BMI and plicometry should be used to assess cardiovascular risk in school-aged children. Further study of these variables in even younger children is advisable. On the other hand, we found no correlation between cIMT and systolic and diastolic blood pressure, waist circumference, or waist-to-height ratio. The correlation between obesity and CIMT has been demonstrated previously by Gazolla et al. ${ }^{18,19}$ It is important that patients with obesity have appropriate clinical and laboratory follow-up.

As we studied patients with obesity, we expected to find elevated serum leptin levels. The values in our sample were similar to those previously described in the literature, suggesting that our patients resembled those in other populations ${ }^{15}$. However, a significant relationship between leptin levels and cIMT could not be demonstrated in our sample, whereas Beauloye et al. found an inverse correlation between cIMT and adiponectin levels ${ }^{20}$.

The study did not demonstrate any correlation between cIMT and leptin or the other laboratory parameters we studied. Our findings suggest that evaluating anthropometric variables, rather than biochemical data are important for detecting elevated cardiovascular risk in children.

The population we studied tends to certain eating habits that condition an altered metabolic behavior as seen in more extensive studies, resulting in a reasonably homogeneous population to parameterize the present study. While recognizing that the study is based on a small sample, with the reserves of the case, we present the mentioned conclusions.

Nevertheless, we can determine a paramount perspective of the metabolic behavior that encompasses this study, given that the evaluation of cIMT represents a reliable and noninvasive measure of subclinical atherosclerosis and an optimal indicator of cardiovascular risk ${ }^{21}$. Moreover, the results found correlate a positive and promising resolution aimed at further analysis toward a true index of clinical variables altered in pediatric patients with obesity and cardiovascular subclinical or clinical disease.

The strength of this study is expressed by showing increased cIMT values in the majority of the patients with obesity and also correlating this with outcome parameters such as an increased triceps skinfold. We can further discuss whether the population without increased cIMT values account for a group with less metabolic risk factors or have less chronicity with the metabolic disease. 


\section{Conclusions}

The anthropometric parameters: BMI, and plicometry should continue to be used in the evaluation of cardiovascular risk of the pediatric population with obesity.

\section{Ethical standards}

All blood samples from patients were obtained and managed according to the corresponding ethical standards. Every aspect of the doctor-patient assessment was managed with the corresponding ethical standards.

Keynotes:

- cIMT is a good and early predictor of cardiovascular disease that explains a strong correlation between coronary and carotid atherosclerosis

- Obesity is associated with a high risk of abnormal cIMT. The findings in our study, therefore, suggest that the anthropometric parameters of BMI and plicometry should be used to assess cardiovascular risk in school-aged children and reinforced with a cIMT evaluation by ultrasound

- A substantial number of patients with abnormal cIMT might also present pre-hypertension, hypertension, and high cholesterol levels.

\section{Funding}

The ELISA kit for leptin measurement was paid for by the main researcher. The rest of the materials were available at the Centro de Atención Médica Ambulatoria de la Fundación Santos y de la Garza Evia and in the Laboratory of Biotechnology of the Instituto Tecnológico y de Estudios Superiores de Monterrey. Participation in the study generated no costs for the patients or their families.

\section{Financial support}

This research received no specific grant from any funding agency, commercial, or nonprofit sectors.

\section{Conflicts of interest}

The authors do not have conflicts of interest to declare.

\section{Ethical disclosures}

Protection of human and animal subjects. The authors declare that no experiments were performed on humans or animals for this study.
Confidentiality of data. The authors declare that they have followed the protocols of their work center on the publication of patient data.

Right to privacy and informed consent. The authors have obtained the written informed consent of the patients or subjects mentioned in the article. The corresponding author is in possession of this document.

\section{References}

1. Klish WJ. Clinical Evaluation of the Obese Child and Adolescent. In: UpToDate; 2017.

2. ONU. La Obesidad Infantil Se Ha Multiplicado Por 10 En 40 Años. México Ocupa Primer Lugar; 2017. Available from: http://www.onunoticias. mx/obesidad-infantil-multiplicado.

3. OECD. Obesity Update 2017. 2017.

4. Ávila MH, Domarco JR. Encuesta Nacional De Salud Y Nutrición De Medio Camino. México: Fecha de publicación; 2017.

5. Urbina EM, Kimball TR, McCoy CE, Khoury PR, Daniels SR, Dolan LM, et al. Youth with obesity and obesity-related Type 2 diabetes mellitus demonstrate abnormalities in carotid structure and function. Circulation. 2009;119:2913-9.

6. Tounian P, Aggoun Y, Dubern B, Varille V, Guy-Grand B, Sidi D, et al. Presence of increased stiffness of the common carotid artery and endothelial dysfunction in severely obese children: a prospective study. Lancet. 2001;358:1400-4.

7. Freedman DS, Dietz WH, Tang R, Mensah GA, Bond MG, Urbina EM, et al. The relation of obesity throughout life to carotid intima-media thickness in adulthood: the bogalusa heart study. Int $\mathrm{J}$ Obes Relat Metab Disord. 2004;28:159-66.

8. de Groot E, Kastelein JJ. Carotid Intima-Media Thickness. In: UpToDate. 2017.

9. Iannuzzi A, Licenziati MR, Acampora C, Salvatore V, Auriemma L, Romano ML, et al. Increased carotid intima-media thickness and stiffness in obese children. Diabetes Care. 2004;27:2506-8.

10. Tarzamni MK, Salehi R, Givian F, Farhang S. Association of carotid intima-media thickness with the presence and severity of coronary artery disease. Neurosciences (Riyadh). 2006;11:308-11.

11. Doyon A, Kracht D, Bayazit AK, Deveci M, Duzova A, Krmar RT, et al. Carotid artery intima-media thickness and distensibility in children and adolescents: reference values and role of body dimensions. Hypertension. 2013;62:550-6.

12. de Onis M, Habicht JP. Anthropometric reference data for international use: recommendations from a world health organization expert committee. Am J Clin Nutr. 1996;64:650-8.

13. National High Blood Pressure Education Program Working Group on High Blood Pressure in Children and Adolescents. The fourth report on the diagnosis, evaluation, and treatment of high blood pressure in children and adolescents. Pediatrics. 2004;114:555-76.

14. Valle Jiménez $M$, Martos $R$, Morales $R M$, Valle $R$, Cañete $M D$ Urbano MM, et al. Relationship between changes in plasma leptin concentrations and plasminogen activator inhibitor-1 in obese prepubertal children after nine months of treatment. Ann Nutr Metab. 2013; 63:216-22.

15. Kahl L, Hughes $H$. The Harriet Lane Handbook. $21^{\text {st }}$ ed. Philadelphia, PA: Elsevier; 2017. p. 1272.

16. American Academy of Pediatrics. Cholesterol Levels in Children and Adolescents. US: American Academy of Pediatrics; 2015.

17. Silva LR, Stefanello JMF, Pizzi J, Timossi LS, Leite N. Aterosclerose subclínica e marcadores inflamatórios em crianças e adolescentes obesos e não obesos. Rev Bras Epidemiol. 2012;15:804-16.

18. Gazolla FM, Neves Bordallo MA, Madeira IR, de Miranda Carvalho CN, Vieira Monteiro AM, Pinheiro Rodrigues NC, et al. Association between cardiovascular risk factors and carotid intima-media thickness in prepubertal brazilian children. J Pediatr Endocrinol Metab. 2015;28:579-87.

19. Reinehr T, Kiess W, de Sousa G, Stoffel-Wagner B, Wunsch R. Intima media thickness in childhood obesity: relations to inflammatory marker, glucose metabolism, and blood pressure. Metabolism. 2006;55:113-8.

20. Beauloye V, Zech F, Tran HT, Clapuyt P, Maes M, Brichard SM, et al. Determinants of early atherosclerosis in obese children and adolescents. $\mathrm{J}$ Clin Endocrinol Metab. 2007;92:3025-32.

21. Farello G, Antenucci A, Stagi S, Mazzocchetti C, Ciocca F, Verrotti A, et al. Metabolically healthy and metabolically unhealthy obese children both have increased carotid intima-media thickness: a case control study. BMC Cardiovasc Disord. 2018;18:140. 